PROCEEDINGS OF THE

AMERICAN MATHEMATICAL SOCIETY

Volume 127, Number 1, January 1999, Pages 29-33

S $0002-9939(99) 04677-8$

\title{
A COMBINATORIAL FORMULA OF LEIBNIZ TYPE WITH APPLICATION TO GEGENBAUER'S POLYNOMIALS
}

\author{
KATSUNORI IWASAKI AND HIROYUKI KAWAMUKO
}

(Communicated by Hal L. Smith)

\begin{abstract}
We establish a combinatorial formula of Leibniz type, which is an identity for a certain differential polynomial. The formula leads to new quadratic relations between Gegenbauer's orthogonal polynomials.
\end{abstract}

\section{INTRODUCTION AND RESULTS}

In the course of his studies on the fourth Painlevé equation in several variables [3], the second author came across the following interesting identity:

$$
\sum_{i=0}^{n} \frac{1}{\alpha+i}\left(\begin{array}{c}
n \\
i
\end{array}\right)\left(D^{i} f^{-\alpha-i}\right)\left(D^{n-i} f^{\alpha+i}\right)=0 \quad(n=1,2,3, \ldots)
$$

where $\alpha$ is a parameter, $f$ is any function in one variable $x$ and $D$ is the differentiation with respect to $x$. He checked by the computer algebra system REDUCE that (1.1) actually holds for $n=1,2, \ldots, 10$. This experiment naturally convinced him that (1.1) should hold for any positive integer $n$. Afterwards the first author gave a proof of this conjecture, which we will present in this paper. The precise meaning of (1.1) is this: the left-hand side of (1.1) is expressed as $f^{-n} F_{n}\left(f, f^{\prime}, \ldots, f^{(n)}, \alpha\right)$ for some polynomial $F_{n}\left(X_{0}, X_{1}, \ldots, X_{n}, Y\right) \in \mathbb{Z}\left[X_{0}, X_{1}, \ldots, X_{n}, Y\right]$ (see Lemma 2.2), and (1.1) asserts that $F_{n}=0$ as a polynomial over $\mathbb{Z}$.

It should be noted that a slightly different identity:

$$
\sum_{i=0}^{n}\left(\begin{array}{c}
n \\
i
\end{array}\right)\left(D^{i} f^{-\alpha}\right)\left(D^{n-i} f^{\alpha}\right)=0 \quad(n=1,2,3, \ldots),
$$

can quite easily be established by using Leibniz's formula. Indeed, the left-hand side of (1.2) is nothing but $D^{n}\left(f^{-\alpha} f^{\alpha}\right)=0$. As we shall see in this paper, however, the verification of (1.1) requires more difficult arguments.

By taking $f$ to be various functions, the identity (1.1) produces many interesting formulas, only one of which is presented below. Set $f=\left(1-x^{2}\right)^{-1}$ and $\alpha=$ $\nu-1 / 2$. Then after some computations, (1.1) yields the following quadratic relations

Received by the editors May 2, 1997.

1991 Mathematics Subject Classification. Primary 05A19, 33C45, 42C05.

Key words and phrases. Combinatorial formula of Leibniz type, Gegenbauer's polynomials, transcendency of the Gamma function. 
between Gegenbauer's polynomials (see e.g. [1] for their definition):

$$
\sum_{i=0}^{n}(i+2 \nu)_{n-1} C_{i}^{\nu}(x) C_{n-i}^{1-n-\nu}(x)=0 \quad(n=1,2,3, \ldots),
$$

where $(\alpha)_{0}=1$ and $(\alpha)_{n}=\alpha(\alpha+1) \cdots(\alpha+n-1)$ for any positive integer $n$. Finally we refer to [3] for another application of (1.1) to the Hamiltonian structure of the fourth Painlevé equation in several variables.

\section{Proof}

Lemma 2.1. Let $P_{n}\left(X_{0}, X_{1}, \ldots, X_{n}, Y\right) \in \mathbb{Z}\left[X_{0}, X_{1}, \ldots, X_{n}, Y\right]$ be inductively defined by $P_{1}\left(X_{0}, X_{1}, Y\right)=X_{1}$ and

$$
\begin{array}{r}
P_{n+1}\left(X_{0}, X_{1}, \ldots, X_{n+1}, Y\right)=(Y-n) X_{1} P_{n}\left(X_{0}, X_{1}, \ldots, X_{n}, Y\right) \\
+X_{0} \sum_{i=0}^{n} X_{i+1} \frac{\partial P_{n}}{\partial X_{i}}\left(X_{0}, X_{1}, \ldots, X_{n}, Y\right) \\
(n=1,2,3, \ldots) .
\end{array}
$$

Then $P_{n}$ is of degree $n-1$ with respect to $Y$ and weighted homogeneous of degree $n$ with respect to $X_{0}, X_{1}, \ldots, X_{n}$, where $\operatorname{deg} X_{i}=i$. Moreover,

$$
D^{n} f^{\alpha}=\alpha f^{\alpha-n} P_{n}\left(f, f^{\prime}, \ldots, f^{(n)}, \alpha\right) \quad(n=1,2,3, \ldots) .
$$

Proof. The first part of the lemma is easy to see. We show (2.1) by induction on $n$. It is clear that (2.1) holds for $n=1$. Assume that (2.1) holds for $n$. Then we have

$$
\begin{aligned}
D^{n+1} f^{\alpha} & =D\left(D^{n} f^{\alpha}\right)=\alpha D\left\{f^{\alpha-n} P_{n}\left(f, f^{\prime}, \ldots, f^{(n)}, \alpha\right)\right\} \\
& =\alpha(\alpha-n) f^{\alpha-n-1} f^{\prime} P_{n}\left(f, f^{\prime}, \ldots, f^{(n)}, \alpha\right) \\
& +\alpha f^{\alpha-n} D P_{n}\left(f, f^{\prime}, \ldots, f^{(n)}, \alpha\right) \\
& =\alpha f^{\alpha-(n+1)}\left\{(\alpha-n) f^{\prime} P_{n}\left(f, f^{\prime}, \ldots, f^{(n)}, \alpha\right)\right. \\
& \left.+f \sum_{i=0}^{n} f^{(i+1)} \frac{\partial P_{n}}{\partial X_{i}}\left(f, f^{\prime}, \ldots, f^{(n)}, \alpha\right)\right\} \\
& =\alpha f^{\alpha-(n+1)} P_{n+1}\left(f, f^{\prime}, \ldots, f^{(n+1)}, \alpha\right) .
\end{aligned}
$$

Hence (2.1) remains true for $n+1$. Thereby the induction is complete.

Lemma 2.2. The left-hand side of (1.1) is expressed as $f^{-n} F_{n}\left(f, f^{\prime}, \ldots, f^{(n)}, \alpha\right)$, where $F_{n}\left(X_{0}, X_{1}, \ldots, X_{n}, Y\right) \in \mathbb{Z}\left[X_{0}, X_{1}, \ldots, X_{n}, Y\right]$ is given by $F_{1}\left(X_{0}, X_{1}, Y\right)=0$ and

$$
\begin{array}{r}
F_{n}\left(X_{0}, \ldots, X_{n}, Y\right)=P_{n}\left(X_{0}, \ldots, X_{n}, Y\right)-P_{n}\left(X_{0}, \ldots, X_{n},-Y-n\right) \\
-\sum_{i=1}^{n-1}(Y+i)\left(\begin{array}{c}
n \\
i
\end{array}\right) P_{i}\left(X_{0}, \ldots, X_{i},-Y-i\right) P_{n-i}\left(X_{0}, \ldots, X_{n-i}, Y+i\right) \\
(n=2,3,4, \ldots) .
\end{array}
$$

Proof. Apply Lemma 2.1 to the left-hand side of (1.1). 
Lemma 2.3.

$$
\sum_{i=0}^{n}(-1)^{i}\left(\begin{array}{c}
n \\
i
\end{array}\right)[\alpha+i]_{k}=0 \quad(k=0,1, \ldots, n-1),
$$

where $[\alpha]_{0}=1$ and $[\alpha]_{k}=\alpha(\alpha-1)(\alpha-2) \cdots(\alpha-k+1)$ for any positive integer $k$.

Proof. Consider the function $u(x)=x^{\alpha}(1-x)^{n}$. The binomial theorem yields

$$
u(x)=\sum_{i=0}^{n}(-1)^{i}\left(\begin{array}{c}
n \\
i
\end{array}\right) x^{\alpha+i} .
$$

Differentiating this $k$-times, we have

$$
D^{k} u=\sum_{i=0}^{n}(-1)^{i}\left(\begin{array}{c}
n \\
i
\end{array}\right)[\alpha+i]_{k} x^{\alpha+i-k} .
$$

Since $u$ has a zero of order $n$ at $x=1$, we have

$$
\left.D^{k} u\right|_{x=1}=0 \quad(k=0,1, \ldots, n-1) .
$$

Substituting $x=1$ in (2.2) and using (2.3), we establish Lemma 2.3 .

\section{Lemma 2.4 .}

$$
g_{n}(\alpha):=\sum_{i=0}^{n} \frac{(-1)^{i}}{\alpha+i}\left(\begin{array}{c}
n \\
i
\end{array}\right) f^{-\alpha-i}\left(D^{n} f^{\alpha+i}\right)=0 \quad(n=1,2,3, \ldots) .
$$

Proof. In view of Lemma 2.1, we set $p_{n}(\alpha)=P_{n}\left(f, f^{\prime}, \ldots, f^{(n)}, \alpha\right)$. Since $p_{n}(\alpha)$ is of degree $n-1$ with respect to $\alpha$, it can be expressed as

$$
p_{n}(\alpha)=\sum_{k=0}^{n-1} p_{n, k}[\alpha]_{k},
$$

where $p_{n, k}$ are polynomials of $f, f^{\prime}, \ldots, f^{(n)}$. Then it follows that

$$
\begin{aligned}
g_{n}(\alpha) & =\sum_{i=0}^{n} \frac{(-1)^{i}}{\alpha+i}\left(\begin{array}{c}
n \\
i
\end{array}\right) f^{-\alpha-i} \cdot(\alpha+i) f^{\alpha+i-n} p_{n}(\alpha+i) \\
& =f^{-n} \sum_{i=0}^{n}(-1)^{i}\left(\begin{array}{c}
n \\
i
\end{array}\right) \sum_{k=0}^{n-1} p_{n, k}[\alpha+i]_{k} \\
& =f^{-n} \sum_{k=0}^{n-1} p_{n, k}\left\{\sum_{i=0}^{n}(-1)^{i}\left(\begin{array}{c}
n \\
i
\end{array}\right)[\alpha+i]_{k}\right\}=0,
\end{aligned}
$$

where Lemma 2.1, (2.4) and Lemma 2.3 are used to obtain the first, second and fourth equalities, respectively. The proof is complete.

Lemma 2.5. Let $I$ be an open interval in $\mathbb{R}$. For any $\mathbb{C}$-valued, smooth, nowhere vanishing function $f \in C^{\infty}(I)$ and $\alpha \in \mathbb{C} \backslash\{0,-1,-2, \ldots\}$, the identity (1.1) holds in $C^{\infty}(I)$.

Proof. Let $\langle\cdot, \cdot\rangle: C^{\infty}(I) \times C_{0}^{\infty}(I) \rightarrow \mathbb{C}$ be the pairing defined by

$$
\langle f, \phi\rangle=\int_{I} f(x) \phi(x) d x,
$$


where $C_{0}^{\infty}(I)$ is the set of all smooth functions in $I$ with compact support. We consider $\left\langle\left(D^{i} f^{-\alpha-i}\right)\left(D^{n-i} f^{\alpha+i}\right), \phi\right\rangle$. Integration by parts yields

$$
\begin{aligned}
\left\langle\left(D^{i} f^{-\alpha-i}\right)\left(D^{n-i} f^{\alpha+i}\right), \phi\right\rangle & =\left\langle D^{i} f^{-\alpha-i},\left(D^{n-i} f^{\alpha+i}\right) \phi\right\rangle \\
& =-\left\langle D^{i-1} f^{-\alpha-i}, D\left\{\left(D^{n-i} f^{\alpha+i}\right) \phi\right\}\right\rangle \\
& =\cdots=(-1)^{i}\left\langle f^{-\alpha-i}, D^{i}\left\{\left(D^{n-i} f^{\alpha+i}\right) \phi\right\}\right\rangle .
\end{aligned}
$$

Applying Leibniz's formula to $D^{i}\left\{\left(D^{n-i} f^{\alpha+i}\right) \phi\right\}$, we obtain

$$
\left\langle\left(D^{i} f^{-\alpha-i}\right)\left(D^{n-i} f^{\alpha+i}\right), \phi\right\rangle=(-1)^{i} \sum_{j=0}^{i}\left(\begin{array}{l}
i \\
j
\end{array}\right)\left\langle f^{-\alpha-i}\left(D^{n-j} f^{\alpha+i}\right), D^{j} \phi\right\rangle .
$$

Let $h$ denote the left-hand side of (1.1). Then (2.5) yields

$$
\begin{aligned}
\langle h, \phi\rangle & =\sum_{i=0}^{n} \frac{1}{\alpha+i}\left(\begin{array}{c}
n \\
i
\end{array}\right)\left\langle\left(D^{i} f^{-\alpha-i}\right)\left(D^{n-i} f^{\alpha+i}\right), \phi\right\rangle \\
& =\sum_{i=0}^{n} \frac{(-1)^{i}}{\alpha+i}\left(\begin{array}{c}
n \\
i
\end{array}\right) \sum_{j=0}^{i}\left(\begin{array}{c}
i \\
j
\end{array}\right)\left\langle f^{-\alpha-i}\left(D^{n-j} f^{\alpha+i}\right), D^{j} \phi\right\rangle \\
& =\sum_{j=0}^{n}\left\langle\sum_{i=j}^{n} \frac{(-1)^{i}}{\alpha+i}\left(\begin{array}{c}
n \\
i
\end{array}\right)\left(\begin{array}{c}
i \\
j
\end{array}\right) f^{-\alpha-i}\left(D^{n-j} f^{\alpha+i}\right), D^{j} \phi\right\rangle \\
& =\sum_{j=0}^{n}\left(\begin{array}{c}
n \\
j
\end{array}\right)\left\langle\sum_{i=j}^{n} \frac{(-1)^{i}}{\alpha+i}\left(\begin{array}{c}
n-j \\
i-j
\end{array}\right) f^{-\alpha-i}\left(D^{n-j} f^{\alpha+i}\right), D^{j} \phi\right\rangle \\
& =\sum_{j=0}^{n}(-1)^{j}\left(\begin{array}{c}
n \\
j
\end{array}\right)\left\langle\sum_{i=0}^{n-j} \frac{(-1)^{i}}{(\alpha+j)+i}\left(\begin{array}{c}
n-j \\
i
\end{array}\right) f^{-(\alpha+j)-i}\left(D^{n-j} f^{(\alpha+j)+i}\right), D^{j} \phi\right\rangle \\
& =\sum_{j=0}^{n}(-1)^{j}\left(\begin{array}{c}
n \\
j
\end{array}\right)\left\langle g_{n-j}(\alpha+j), D^{j} \phi\right\rangle,
\end{aligned}
$$

where $g_{n}(\alpha)$ is defined in Lemma 2.4. Since $g_{n-j}(\alpha+j)=0$ for $j=0,1, \ldots, n-1$ (see Lemma 2.4) and $g_{0}(\alpha+n)=1 /(\alpha+n)$, we have

$$
\langle h, \phi\rangle=\frac{(-1)^{n}}{\alpha+n} \int_{I}\left(D^{n} \phi\right) d x=\frac{(-1)^{n}}{\alpha+n}\left[D^{n-1} \phi\right]_{I}=0 .
$$

Hence $\langle h, \phi\rangle=0$ for any $\phi \in C_{0}^{\infty}(I)$. The fundamental lemma in variational theory implies $h=0$. The proof is complete.

Remark 2.6. Lemma 2.5 is restated that for any nowhere vanishing function $f \in$ $C^{\infty}(I)$ and $\alpha \in \mathbb{C} \backslash\{0,-1,-2, \ldots\}$, the identity $F_{n}\left(f, f^{\prime}, \ldots, f^{(n)}, \alpha\right)=0$ holds in $C^{\infty}(I)$, where $F_{n}\left(X_{0}, X_{1}, \ldots, X_{n}, Y\right) \in \mathbb{Z}\left[X_{0}, X_{1}, \ldots, X_{n}, Y\right]$ is defined in Lemma 2.2 .

Proof of (1.1). As is mentioned in Section 1, in order to establish (1.1), it suffices to show that $F_{n}\left(X_{0}, X_{1}, \ldots, X_{n}, Y\right)=0$ in $\mathbb{Z}\left[X_{0}, X_{1}, \ldots, X_{n}, Y\right]$. Choose a transcendental number $\alpha \in \mathbb{C}$ and set $f=\Gamma$, the Gamma function. Let $I$ be an open interval in $\mathbb{R}$ on which $\Gamma$ is nowhere vanishing. Then Lemma 2.5 together with Remark 2.6 implies

$$
F_{n}\left(\Gamma, \Gamma^{\prime}, \ldots, \Gamma^{(n)}, \alpha\right)=0
$$


On the other hand, O. Hölder [2] proved that the Gamma function does not satisfy any algebraic differential equation. To be more precise, $\Gamma, \Gamma^{\prime}, \ldots, \Gamma^{(n)}, \ldots$ are algebraically independent over the field $\mathbb{C}(x)$. In particular, if $\alpha$ is a transcendental number, then $\Gamma, \Gamma^{\prime}, \ldots, \Gamma^{(n)}$ and $\alpha$ are algebraically independent over $\mathbb{Z}$. Therefore (2.6) implies that $F_{n}\left(X_{0}, X_{1}, \ldots, X_{n}, Y\right)=0$ in $\mathbb{Z}\left[X_{0}, X_{1}, \ldots, X_{n}, Y\right]$. The proof is complete.

\section{REFERENCES}

1. A. Erdélyi, et al., Higher transcendental functions, Vol. 1, MacGraw Hill, New York, 1953. MR 84m:3001a

2. O. Hölder, Uber die Eingenschaft der Gammafunction keiner algebraischen Differentialgleichung zu genügen, Math. Ann. 28 (1887), 1-13.

3. H. Kawamuko, Studies on the fourth Painlevé equation in several variables, Ph. D. dissertation, The University of Tokyo, 1997.

Department of Mathematics, Kyushu University, 6-10-1 Hakozaki, Higashi-ku, FUKUOKA 812-8581 JAPAN

E-mail address: iwasaki@math.kyushu-u.ac.jp

Department of Mathematical Sciences, The University of Tokyo, 3-8-1 Komaba, Meguro-Ku, TOKYO 153 Japan

E-mail address: kawam@poisson.ms.u-tokyo.ac.jp

Current address: Department of Mathematics, Mie University, 1515 Kamihama, Tsu 514-8507, Japan 